\title{
Optimization of variable stiffness composites with embedded defects induced by Automated Fiber Placement
}

\author{
Mahdi Arian Nik, Kazem Fayazbakhsh, Damiano Pasini*, Larry Lessard \\ Department of Mechanical Engineering, McGill University, Macdonald Engineering Building, 817 Sherbrooke West, \\ Montreal, QC, Canada H3A 2 K6 \\ *Corresponding author: damiano.pasini@mcgill.ca; Tel: (+1) 514-398-6295; fax: (+1) 514-398-7365
}

\begin{abstract}
Variable stiffness composite laminates can be manufactured using Automated Fiber Placement (AFP) technology. An improvement in structural performance can be achieved by tailoring their material properties in directions that are more favorable to carry loads. During AFP manufacturing, however, the formation of defects, mainly gaps and overlaps, is inevitable. The extent of a defected zone is generally controlled by two sets of parameters: design parameters; and manufacturing parameters. In this work, we investigate how the parameters governing the formation of defects impact the set of optimal solutions for a multi-objective optimization problem, where in-plane stiffness and buckling load are simultaneously maximized. It is found that increasing the number of tows within a course reduces the amount of defected areas, where the course width is kept constant. Furthermore, the amount of defect areas significantly reduces by using a wide course, which has the effect of both increasing the deviation from the designed fiber path and reducing the number of manufacturable designs. The results show that a complete gap strategy shifts the defect-free Pareto front, obtained without considering the effect of defects, towards lower in-plane stiffness and buckling load; on the other hand, a complete overlap strategy shifts the Pareto front towards higher structural properties.
\end{abstract}

Keywords: Multi-objective Optimization; Defects; Gap; Overlap; Automated Fiber Placement. 


\section{Introduction}

Automated Fiber Placement (AFP) is a manufacturing technology that offers great flexibility to build composite laminates with a variety of structural geometries and laminate layups. In particular, complex geometries, such as double curvature surfaces, and non-conventional composites with variable stiffness can be produced by laying down fibers along preferred curvilinear paths within the ply. The structural benefits of variable stiffness laminates are achieved by tailoring the material properties in directions that are more favourable to carry loads within the laminates.

The advantage of using curvilinear fibers to improve the structural performance of a composite laminate has been extensively demonstrated [1-6]. Several studies proposed the optimum fiber path that minimizes the compliance of a cantilever beam [7], maximizes the buckling load of a plate [8], maximizes the buckling load of a hybrid composite shell [9], simultaneously maximizes the buckling load and in-plane stiffness [10]. These works are promising because they demonstrate the structural improvement that tailored curvilinear fiber paths can potentially generate. However, these results are only theoretically optimum, as they do not consider the manufacturing constraints, e.g. the minimum turning radius of the fiber path imposed by an AFP machine. As a result, some of the optimum solutions might not be manufacturable. Alhajahmad et al. [11] accounted for the minimum turning radius in the search for the optimal fiber path that maximizes the buckling load of a plate subjected to pressure and in-plane loads. Furthermore, Blom et al. [12] obtained a fiber path that maximizes load-carrying capability of a cylinder under pure bending load.

The above works assume the laminates to be defect-free (ignoring the presence of defects). In practice, however, the method used by an AFP machine to manufacture a laminate with curvilinear fibers generally leads to the formation of defects in the form of gaps and/or overlaps. During the manufacturing process, the first course (a band of tows) is laid down along the designed fiber path. Subsequently, the first course is repeatedly shifted to cover the whole laminate. If the course width could change continuously, there would not be any defects in the laminate. Since an AFP machine can change the course width only by a discrete value via either adding or dropping tows, small defect areas form within the laminate. Triangular gaps and/or overlaps generally appear between adjacent courses, an AFP process outcome that affects the structural performance of the final laminate. There are several strategies to drop the tows. 0\% coverage (complete gap) is a strategy that involves dropping a tow as soon as one edge of the tow reaches a course boundary; it creates 
small triangular areas without fibers, i.e. gaps. Another method is 100\% coverage (complete overlap); here, a tow is dropped when both edges of the tow cross the course boundary, thereby creating small areas of triangular overlaps. An intermediate scenario is when the coverage is between $0 \%$ and $100 \%$ [13]. The strategies explained above can be also followed to add a tow. Another strategy to manufacture a laminate without any gap is to avoid dropping the tows, which results in large overlap areas within the manufactured laminate. This strategy is referred to as the tow-overlapping method.

In the literature, the study of the effect of defects, mainly overlaps, on the mechanical properties of variable stiffness laminates manufactured by AFP has attracted the attention of several researchers. Wu et al. [14] conducted experiments that showed the prebuckling stiffness of a 20-layered variable stiffness panel with tow-overlapping is $27 \%$ higher than a $[ \pm 45]_{5 S}$ cross-ply laminate, while it is only $4 \%$ higher for the panel with gaps. Lopes et al. [15] investigated the first-ply failure load of a variable stiffness laminate. They found that the optimum variable stiffness design obtained by Tatting et al. $[13,16]$ increased the first-ply failure load by $24.8 \%$ and $33.9 \%$ for a complete gap and tow-overlapping strategies, respectively. In another attempt, Lopes et al. [17] further extended the previous results to account for the progressive damage behavior and final structural failure. It was shown that for a flat plate without a central hole, the optimum variable stiffness design can increase the strength by $25.2 \%$ and $41.2 \%$ for a complete gap and tow-overlapping strategies, respectively. The increase in strength for the plate with a central hole is $13.4 \%$ for a complete gap and $55.5 \%$ for a tow-overlapping strategy. It should be noted that in the previously mentioned works gaps are not considered in the analysis of the variable stiffness laminates. Blom et al. [18] first investigated the influence of gaps on strength and stiffness of variable stiffness laminates using the Finite Element Method (FEM). They found that increasing the gap areas in the laminate deteriorates both strength and stiffness properties. They also investigated the effect of tow width on the stiffness and strength of a variable stiffness laminate and concluded that in addition to the size of the gap areas, their distribution also has an effect on the structural properties of the laminate.

The area percentage of gaps and overlaps depends on two sets of parameters: manufacturing parameters, such as the tow width, the number of tows in a course, and the tow drop strategy; and design parameters, which define a curvilinear fiber path. In the past, these parameters were not considered in the optimization of a variable stiffness laminate. On the other hand, one expects that 
optimum fiber paths for an ideal defect-free laminate deviate from those for real manufactured laminates with embedded defects. Hence the aim of this paper is to factor in the effect of gaps and overlaps in the optimization of variable stiffness laminates. In this work, MATLAB subroutines are first developed to calculate the area and extent of gaps and overlaps in a variable stiffness laminate considering both manufacturing and design parameters. Then, the effects of these defects are considered in the calculation of the laminate in-plane stiffness and buckling load. Finally, the optimization problem of maximizing simultaneously in-plane stiffness and buckling load for laminates with embedded defects is formulated and the set of optimal solutions is obtained. A discussion on the effect of design and manufacturing parameters on the optimized variable stiffness designs is presented before the concluding remarks.

\section{Fiber path definition}

A variable stiffness laminate can be designed by defining a reference fiber path along which the AFP machine places the first course. The entire laminate can be manufactured by shifting the reference fiber path perpendicular to the direction of the fiber angle variation. As a reference fiber path, we consider here a constant curvature path presented by Blom et al. [18]. Along this reference path, the fiber orientation can be obtained as

$$
\cos \theta=\cos T_{0}+\frac{|x|}{\rho},
$$

where $\theta$ is the fiber orientation along the fiber path, $T_{0}$ is the fiber orientation at the plate center, and $\rho$ is the turning radius. The fiber orientation varies between $T_{0}$ (at the plate center, $x=0$ ) and $T_{1}$ (at the plate edges, $x= \pm \frac{w}{2}$ ), where the radius of the path remains constant (Figure 1a). To manufacture the entire plate, the reference fiber path should be shifted along the $y$-direction since the fiber orientation varies along the $x$-direction (Figure 1b). A single layer with this fiber path definition may be represented by $\left[+<T_{0} \mid T_{1}>\right.$ ], where $T_{1}=T_{0}$ represents a case of straight-fiber layer [18]. It should be noted that in this study a minimum turning radius of $0.635 \mathrm{~m}$ (25in) is considered as a manufacturing constraint imposed by a typical AFP machine. 


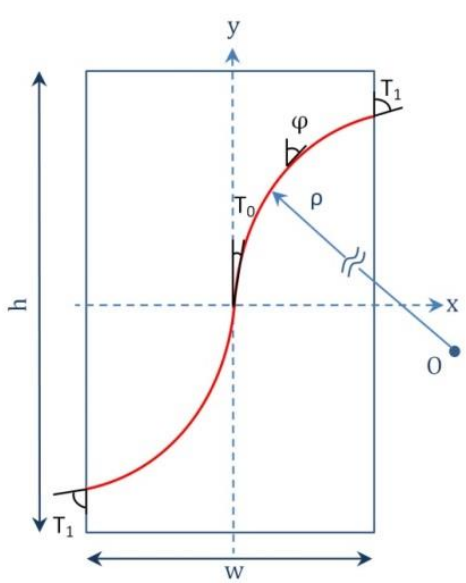

(a)

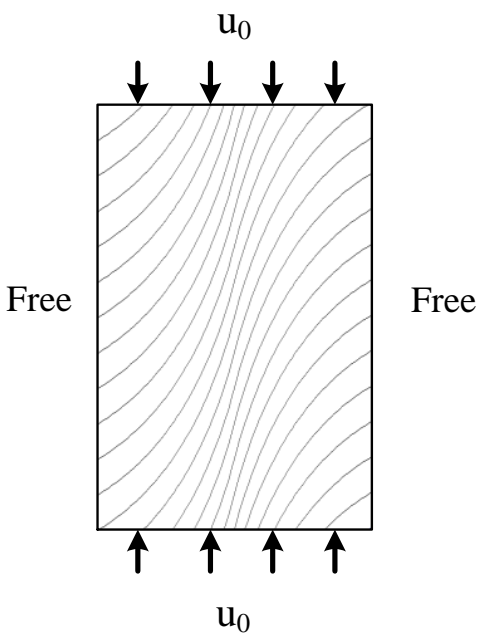

(b)

Figure 1: A composite lamina with a constant curvature fiber path; (a) definition of the reference fiber path; (b) the reference fiber path is shifted along y-direction.

\subsection{Test problem}

A $0.254 \times 0.406 \mathrm{~m}(10 \times 16 \mathrm{in})$ rectangular plate made of 16-ply balanced symmetric laminate $[ \pm \theta(x)]_{4 S}$ subjected to a uniform end shortening along the y-direction is considered as a case study. Concerning the boundary conditions, the transverse edges are considered free (Figure 1b) for in-plane displacement and all edges are simply supported against out-of-plane movement. Carbon epoxy Cytec® G40-800/5276-1 material properties used in this study are as follows: $E_{x}=143 G P a$, $E_{y}=9.1 G P a, G=4.8 G P a$, and $v=0.3$.

\section{Design Parameters}

In Section 2, the reference fiber path for manufacturing a variable stiffness laminate is defined. During manufacturing, the AFP machine head follows the reference fiber path and places the first course, whose centerline exactly matches the reference fiber path. Then, the machine head is shifted to place the subsequent courses. The shift distance is generally chosen to avoid the formation of major gaps or overlaps within the laminate [18]. MATLAB subroutines are developed here to calculate the shift distance, as well as the location, shape, and pattern of gaps and overlaps emerging within the laminate from the AFP manufacturing process [19]. With reference to the shift distance, course boundaries can be obtained based on one-sided or both-sided tow drop approaches. When 
using a one-sided tow drop approach, one boundary of the course is kept parallel to the reference fiber path and the other one is obtained by shifting the first boundary; as a result, gaps and/or overlaps only appear along one of the course boundaries. In a both-sided tow drop approach, both course boundaries are modified and defined by shifting the reference fiber path; therefore, gaps and/or overlaps appear along both course boundaries. Using the one-sided tow drop approach results in a significantly lower gap and/or overlap area percentage within the laminate compared to the both-sided approach. Figure 2 shows the gap (shaded area) distribution within the $[+<45 \mid 26>]$ lamina and compares the two approaches for 8 tows, each $3.175 \mathrm{~mm}(1 / 8 \mathrm{in})$ wide inside each course.

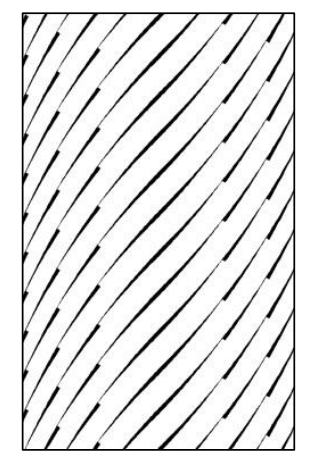

(a)

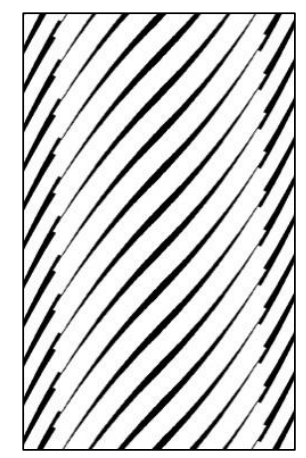

(b)

Figure 2. Gap distribution for the $[+<45 \mid 26>$ ] lamina; (a) one-sided tow drop (gap areas=10.4\%); (b) both-sided tow drop (gap areas $=19.9 \%)$.

It should be noted that the gap area percentage is calculated as the sum of gap area divided by the total laminate area. It can be seen that for this design, the both-sided tow drop approach results in a gap area percentage of $19.9 \%$, which is about twice as much as the gap area percentage that would appear by using the one-sided two drop approach (10.4\%). Moreover, the one-sided tow drop approach results in more scattered gap areas compared to the both-sided two drop approach.

Besides the tow drop approach, the gap and overlap area percentages depend on the design parameters $\left(T_{0}\right.$ and $\left.T_{1}\right)$, which define the fiber path. Figure 3 shows the gap and overlap area percentages in laminates with a constant curvature fiber path as a function of $T_{0}$ and $T_{1}$. All manufacturable layups, which are investigated here, assume a one-sided tow drop approach and consider 8 tows with a width of $3.175 \mathrm{~mm}$ within each course. To determine the manufacturable layups, the minimum turning radius of $0.635 \mathrm{~m}$ ( $25 \mathrm{in}$ ) was constrained for the entire course. 


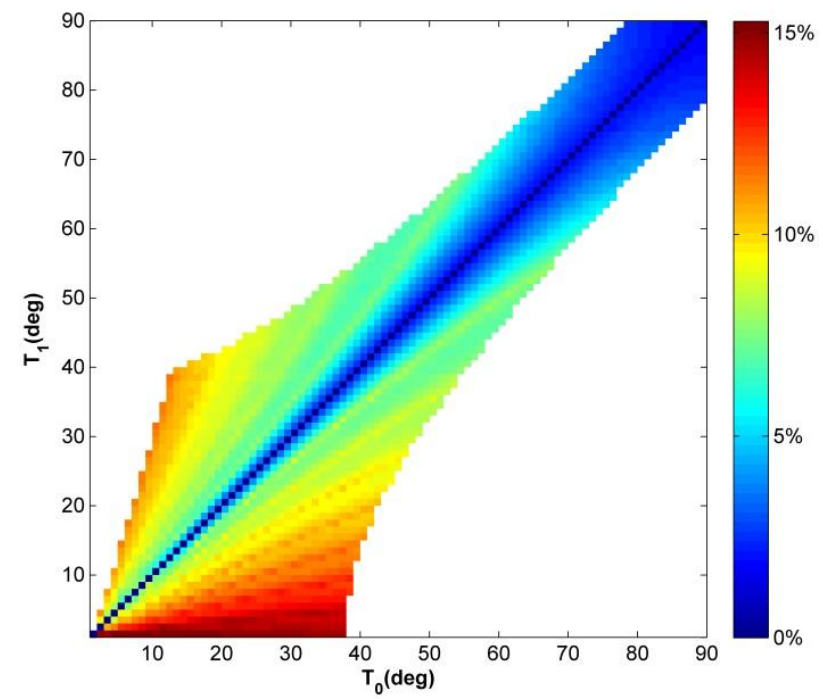

(a)

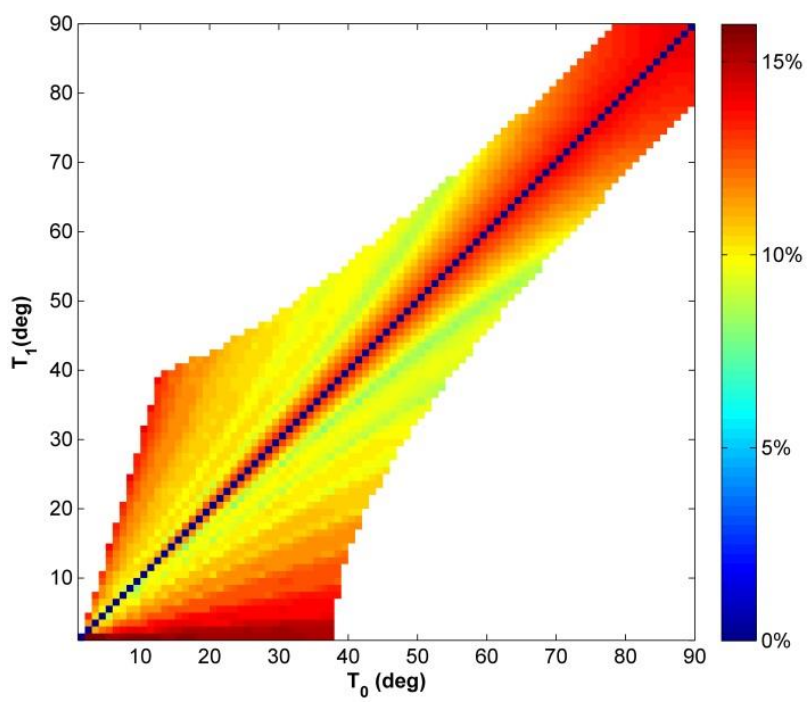

(b)

Figure 3. Defect area percentage as a function of $\mathrm{T}_{0}$ and $\mathrm{T}_{1}$ for laminates with a constant curvature fiber path; (a) overlap area percentage following a complete overlap strategy; (b) gap area percentage following a complete gap strategy.

Figure 3 shows that there are no gaps and overlaps for a straight-fiber case, where $\mathrm{T}_{0}=\mathrm{T}_{1}$. The gap and overlap area percentages increase as the difference between $T_{0}$ and $T_{1}$ becomes larger. The reason is that for a design with a large difference between $T_{0}$ and $T_{1}$, the fiber path has a large curvature, which increases the number of tow drop incidents during the manufacturing. As a result, the gap and overlap area percentages within the laminate increase. In the case of a complete gap strategy (Figure $3 \mathrm{~b}$ ), the gap area percentage is large for the case with a $\mathrm{T}_{0}$ close to $\mathrm{T}_{1}$ as in a complete gap strategy a tow is dropped as soon as one edge of the tow reaches the course boundary; thus, in a design very close to a straight-fiber case (a small difference between $\mathrm{T}_{0}$ and $\mathrm{T}_{1}$ ), a missing tow in each course causes a long and wide gap. It is also found that layups with a large value of $\mathrm{T}_{0}$ and $\mathrm{T}_{1}$ generally have a smaller overlap area percentage than the cases with a small fiber orientation at the plate center or edges.

\section{Manufacturing parameters}

The tow width and the number of tows in a course are manufacturing parameters that have also a significant effect on the gap and overlap area percentages. Tows are a thin bundle of fibers and resin that typically have a width of $3.175 \mathrm{~mm}$ (1/8 in), $6.35 \mathrm{~mm}(1 / 4 \mathrm{in})$, or $12.7 \mathrm{~mm}(1 / 2 \mathrm{in})$. Each tow 
can be fed at its own rate to allow steering for that tow. In addition, each tow can be cut and restarted independently from the other ones, thereby increasing AFP machine's manufacturing flexibility. Depending on the head configuration, $8,12,16,24$, and 32 tows can be laid down sideby-side on the mold. In this section, the effect of these parameters is investigated through a parametric study of five designs on the defect-free (ignoring the presence of defects) Pareto front obtained for simultaneously maximizing in-plane stiffness and buckling load [19]. These designs are selected to yield the maximum Euclidean distance from each other in the design space.

\subsection{The effect of the number of tows}

For the five selected designs, Figure 4 illustrates the effect of the number of tows on the gap area percentage within the laminate considering a complete gap strategy, where the tow width is kept constant at $3.175 \mathrm{~mm}$ (1/8 in). Thus, a larger number of tows in a course represents a wider course.

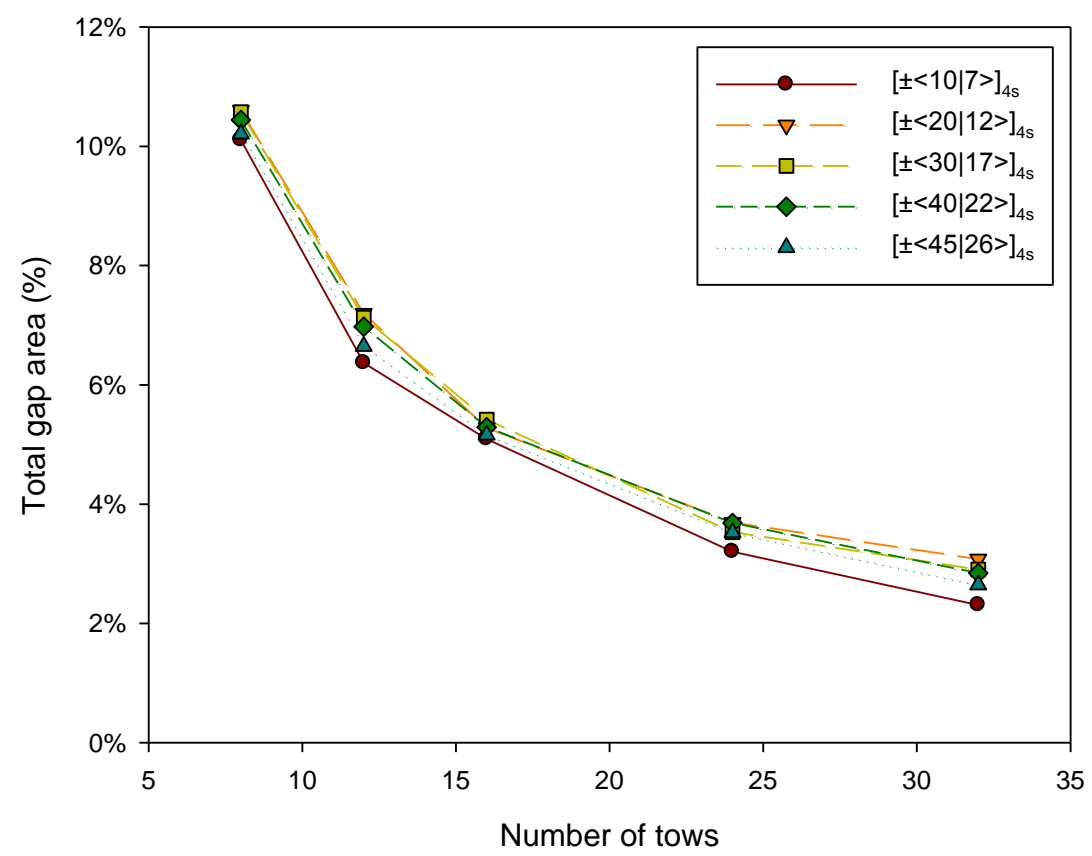

Figure 4. The effect of the number of tows inside a course on the gap area percentage within the laminate (the tow width is kept constant at $3.175 \mathrm{~mm}(1 / 8 \mathrm{in})$.

It is observed that an increase in the number of tows inside a course reduces the gap area percentage within the laminate, where the tow width remains constant. For example, for the $[ \pm<45 \mid 26>]_{4 \mathrm{~S}}$ design, using 8 tows in a course (course width of $25.4 \mathrm{~mm}$ ) results in a total of $10.1 \%$ gap within the laminate, which is about three times more compared to a course with 32 tows (the course width of 
$101.6 \mathrm{~mm}$ ), creating $3.6 \%$ gap. The trend occurs because the use of a large number of tows in a course (a wide course) reduces the number of course boundaries within the laminate, thereby reducing the total number of tow drop incidents, which in turn results in a lower gap area percentage. A similar trend exists for the overlap area percentage within the laminate.

It is worth mentioning that using a wide course reduces the number of manufacturable designs since the minimum turning radius constraint must be satisfied over the entire course rather than the course centerline. Figure 5 shows that a large number of optimum designs obtained by assuming an infinitely small course width are not actually manufacturable with a course width of $101.6 \mathrm{~mm}$ (4 in) since the minimum turning radius is not satisfied over the entire course. Comparing Figures 4 and 5 , it can be seen that a wide course significantly reduces the amount of defects, while it decreases the number of manufacturable designs. Therefore, it is necessary to consider the tow width and the number of tows during the optimization process.

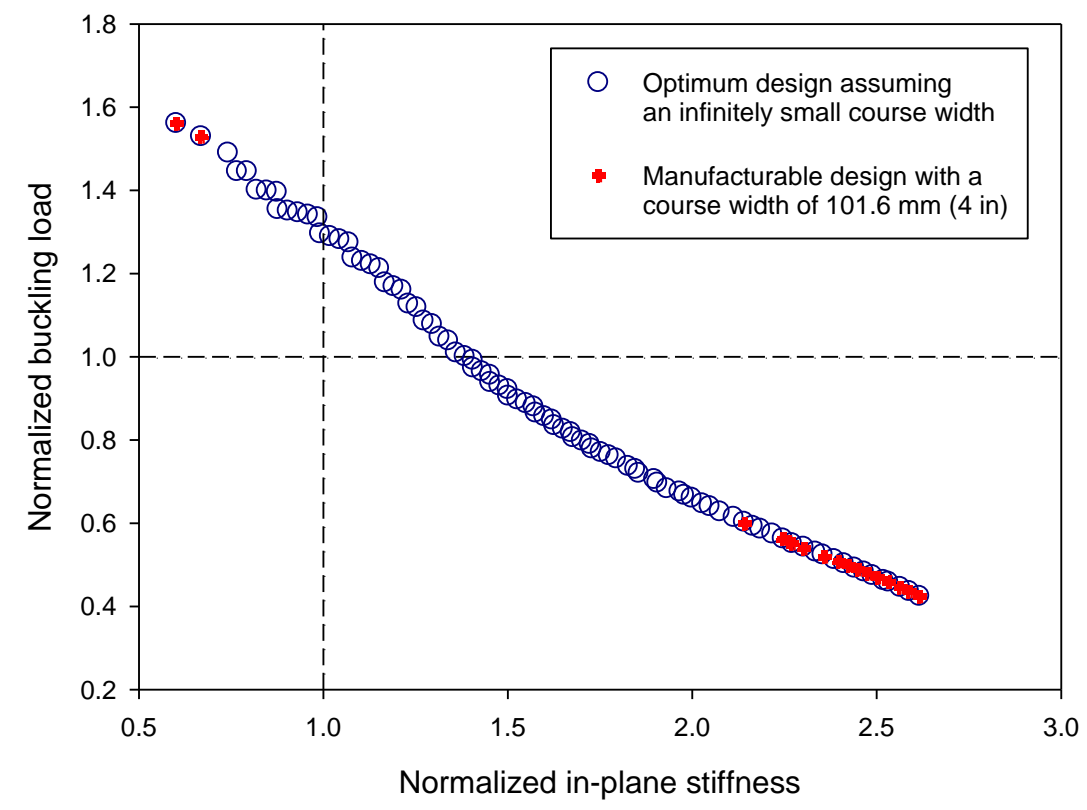

Figure 5. Manufacturable optimum designs with a course width of $101.6 \mathrm{~mm}$ (4 in).

Using a wider course increases the deviation of the fiber angle within the course from the optimized reference fiber path. Figure 6 illustrates the effect of the number of tows on the bucking load for the $[ \pm<45 \mid 26>]_{4 s}$ laminate, where the tow width is kept constant. This design is chosen because it 
offers the maximum buckling load and satisfies the minimum turning radius constraint over the entire course, even for the widest course considered in this study, i.e. $101.6 \mathrm{~mm}$ (4 in). It should be noted that the buckling load is normalized with respect to the corresponding values of a constant stiffness quasi-isotropic laminate with a [45/0/-45/90] 2 S layup, the baseline. In addition, for the case with overlap, the buckling load is divided by the panel weight to account for the additional weight of the overlap areas and to compare the plate's performance on an equal-weight basis.

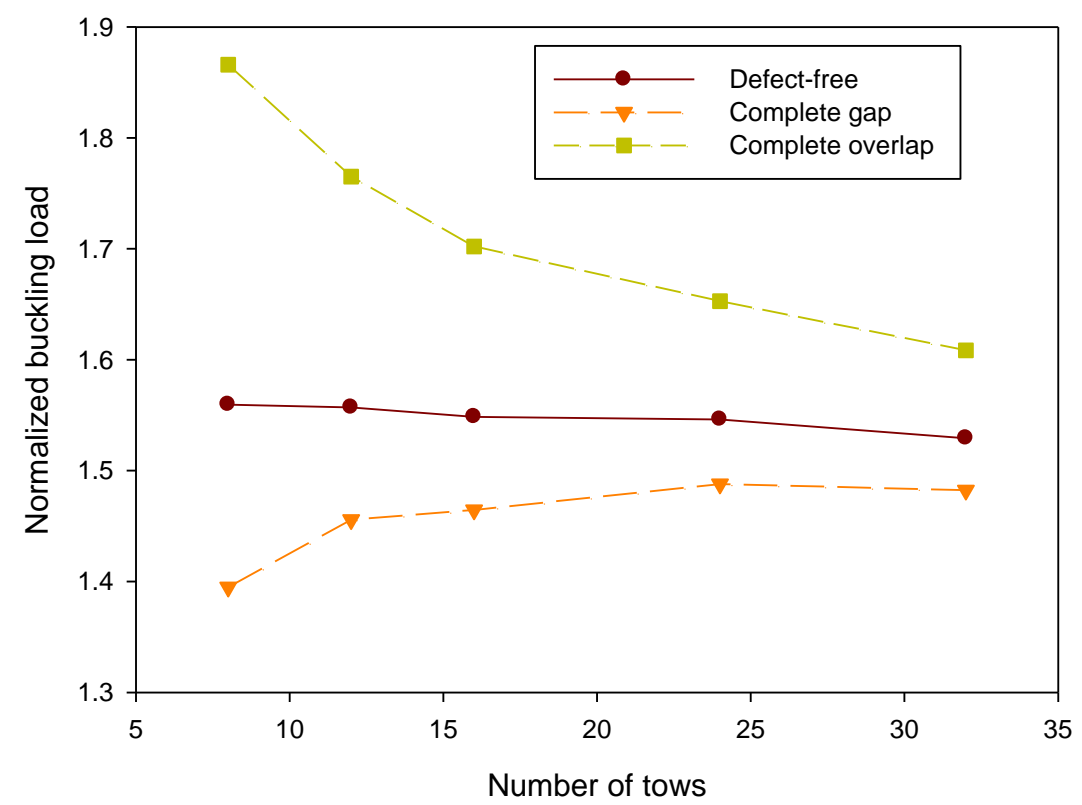

Figure 6. The effect of the number of tows in a course on the buckling load of the $[ \pm<45 \mid 26>]_{4 S}$ laminate. Tow width is kept constant at $3.175 \mathrm{~mm}(1 / 8 \mathrm{in})$;

The defect-free case in Figure 6 isolates the effect of the fiber angle deviation from the effect of defects on the buckling load of the $[ \pm<45 \mid 26>]_{4 s}$ laminate. The defect-free case indicates that generally an increase in the course width (a large number of tows) slightly reduces the buckling load of the laminate. It is because a wide course results in an increase in the deviation of the fiber angle within the course from the centerline, here, the optimized fiber path. On the other hand, the complete gap and overlap cases in Figure 6 show that gap and overlap have a higher impact on the buckling load of a variable stiffness laminate compared to the fiber angle deviation within a course. 


\subsection{The effect of the tow width}

This section investigates the effect of the tow width on the amount of defects within a laminate, where the course width is kept constant at $101.6 \mathrm{~mm}$ (4 in). Figure 7 shows the change in the gap area percentage with respect to the tow width for the five selected designs.

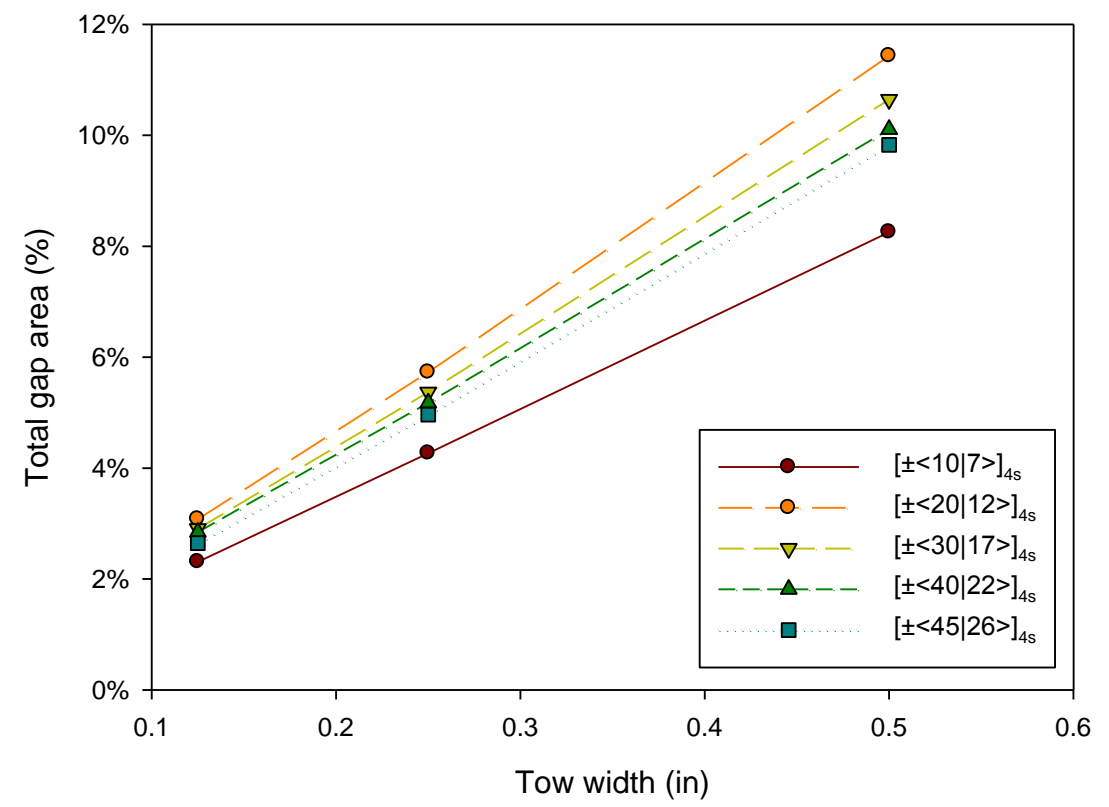

Figure 7. The effect of tow width on the gap area percentage within the laminate (the course width is kept constant at 4 in).

Since the course width is kept constant, the number of course boundaries in the laminate does not change. It is expected that dropping a wider tow results in a larger amount of defects. In such a case, it should be noted that the number of tow drop incidents is smaller than a case with a narrower tow. Figure 7 illustrates that the amount of gap areas within the laminate increases with an increase in the tow width. This trend also exists for overlaps. As an example, for a $[ \pm<20 \mid 12>]_{4 s}$ laminate, using a tow width of $12.7 \mathrm{~mm}(1 / 2 \mathrm{in})$ creates $11.4 \%$ gap, whereas using a tow width of $3.175 \mathrm{~mm}(1 / 8 \mathrm{in})$ results in $3.1 \%$ of gap within a laminate. It can be concluded from Figures 4 and 7 that using the largest number of tows with the smallest width minimizes the gap and overlap area percentages within the laminate. 
Considering all combinations of the manufacturing parameters ( 9 combinations), Figure 8 shows the reduction in the buckling load and in-plane stiffness of the laminates compared to the corresponding values of the defect-free case as a function of the gap area percentage. It can be seen that generally an increase in the gap area percentage results in a larger reduction in the laminate buckling load and in-plane stiffness. Although the gap area percentage is a determining factor on the performance of the laminate, it is not the only one. For example, for a gap area of about $10 \%$, the change in the buckling load varies from $10 \%$ to $12 \%$ depending on the laminates layup (design parameters); the variation range for in-plane stiffness is between $10 \%$ and $14 \%$. These values imply that the design parameters have an effect on the gap distribution within the laminate and should thus be considered along with the manufacturing parameters to optimize the structural performance of a variable stiffness laminate.

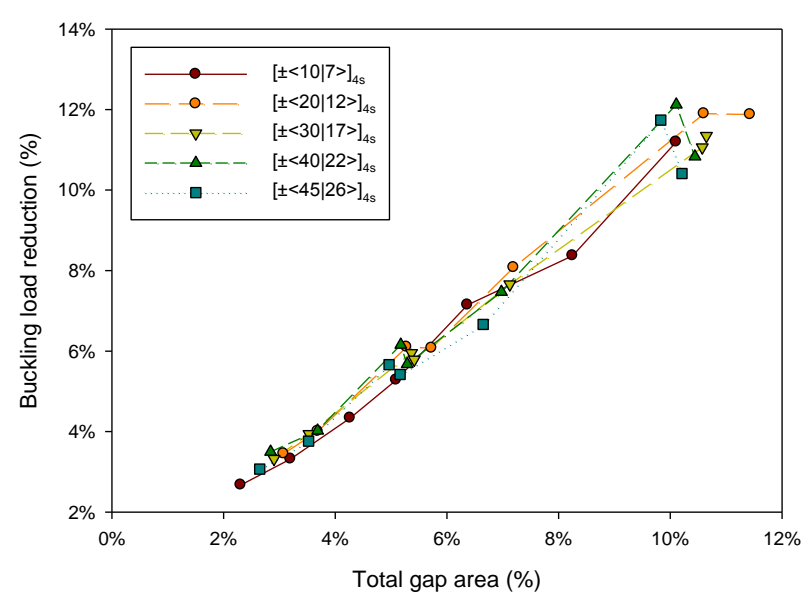

(a)

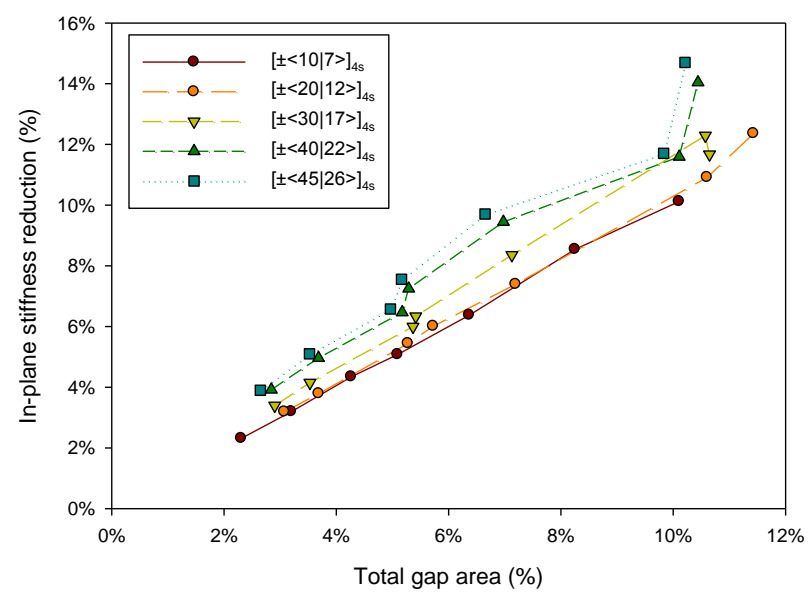

(b)

Figure 8 . The effect of gap area percentage on the structural performance of variable stiffness laminates (a) the bucking load and (b) in-plane stiffness.

\section{Optimization of composite laminates with embedded defects}

Section 3 has shown that the design and manufacturing parameters of a variable stiffness laminate have a significant effect on the gap and overlap area percentages, as well as on its buckling load and in-plane stiffness. To investigate the maximum influence of gaps and overlaps on the optimization results, we examine here manufacturing parameters that produce the maximum amount of defects, 
i.e. 8 tows with a width of $3.175 \mathrm{~mm}(1 / 8 \mathrm{in})$. The goal is to find the optimum fiber path parameters (design variables) of defected laminates for maximum buckling load and in-plane stiffness. One way to convert a maximization problem into a minimization problem is to use the inverse of the objective functions. The optimization problem can be written as follows

$$
\begin{aligned}
& \min _{\mathbf{x}}\left\{1 / E_{e q}(\mathbf{x}), 1 / N_{c r}(\mathbf{x})\right\} ; \mathbf{x}=\left(T_{0}, T_{1}\right)^{T} \\
& \text { s.t. }\left\{T_{0}, T_{1} \in\left[0^{\circ}, 90^{\circ}\right] \& R \geq 25 \text { in }\right\}
\end{aligned}
$$

Where $\mathbf{x}$ is the vector of the design variables, i.e., $T_{0}$ and $T_{1}$, which are the fiber angles at the plate center and the plate edges, respectively (Figure 1a); $E_{e q}$ and $N_{c r}$ are respectively the equivalent inplane stiffness and the buckling load of the plate, and $R$ is the minimum turning radius of the fibers over the entire course width. 


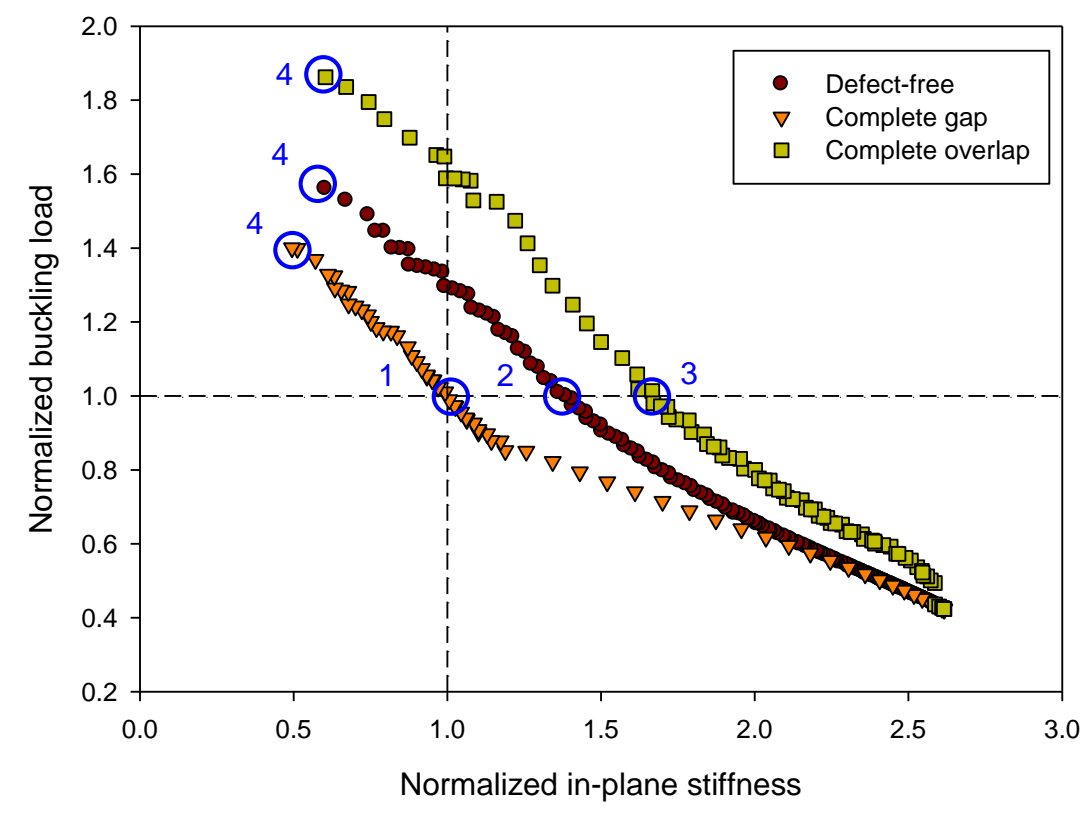

(1)

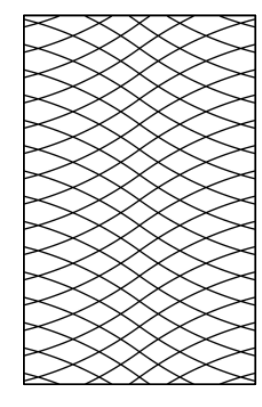

$[ \pm<36 \mid 14>]_{4 S}$
(2)

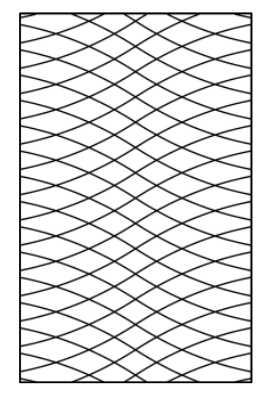

$[ \pm<33 \mid 11>]_{4 S}$
(3)

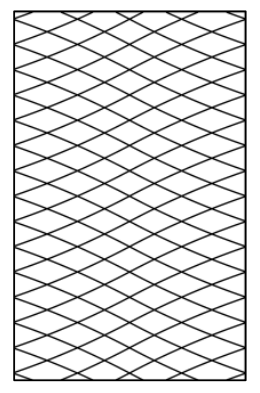

$[ \pm<28 \mid 19>]_{4 S}$
(4)

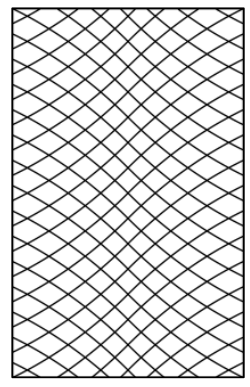

$[ \pm<45 \mid 26>]_{4 S}$

Figure 9. Pareto front obtained considering the effect of gaps and overlaps.

Figure 9 shows that a complete gap strategy shifts the defect-free Pareto front towards lower inplane stiffness and buckling load, whereas a complete overlap strategy shifts it towards higher values of in-plane stiffness and buckling load. Recall that buckling load and in-plane stiffness of variable stiffness laminates are normalized with respect to the values corresponding to a constant stiffness quasi-isotropic laminate with a $[45 / 0 /-45 / 90]_{2 S}$ layup, the baseline. In addition, for the cases with overlaps, the buckling load and in-plane stiffness are divided by the panel weight. 
For all three cases, i.e. the defect-free, the complete gap strategy, and the complete overlap strategy, the $[ \pm<45 \mid 26>]_{4 S}$ design, yields the highest buckling load. It is expected that by assuming a defect-free laminate, this design improves the buckling load by $56 \%$ compared to the baseline, which comes at the cost of $40 \%$ reduction of in-plane stiffness. However, the $[ \pm<45 \mid 26>]_{4 S}$ laminate, manufactured with a complete gap strategy, has only $40 \%$ higher buckling load and $49 \%$ lower in-plane stiffness compared to the baseline. In other words, incorporating the effect of gaps in the design deteriorates both the objectives. On the other hand, the manufactured laminate using a complete overlap strategy has $86 \%$ higher buckling load and $40 \%$ lower in-plane stiffness, which means that, on an equal-weight basis, incorporating the effect of overlaps improves only the buckling load of the laminate.

The difference between the gap and overlap modified Pareto fronts is the largest for the laminates which provide the maximum buckling load improvement, whereas it decreases as the layup becomes closer to the cases that provide the maximum in-plane stiffness. This can be explained as the designs which provide high buckling load have a fiber path with larger curvature and a larger amount of defects compared to the designs which have high in-plane stiffness. The design which has the maximum in-plane stiffness is a straight fiber case with all fibers along the loading direction, an intuitive solution based on the classical laminate theory. The layups of the two anchor points of each Pareto front do not change by considering or ignoring the presence of defects (Figure 9). However, the layups of the other designs on the three Pareto fronts are different from each other. For example, for a defect-free case, the $[ \pm<33 \mid 11>]_{4 S}$ layup provides the same buckling load as the baseline, but higher in-plane stiffness. When gaps are included in the optimization, the $[ \pm<36 \mid 14>]_{4 S}$ laminate has the buckling load of the baseline and higher in-plane stiffness. The $[ \pm<28 \mid 9>]_{4 S}$ laminate has the above characteristic when overlaps are included in the optimization. This demonstrates that the effect of gaps and overlaps needs to be considered in the calculation of the objective functions in the optimization problem, as the optimum designs might be different than those obtained for a defect-free laminate. 


\section{Conclusions}

The effect of design and manufacturing parameters on the gap and overlap area percentages within variable stiffness laminates has been investigated. The buckling load and in-plane stiffness of defected laminates have been calculated and the impact of gaps and overlaps has been depicted on the Pareto solutions of variable stiffness laminate maximizing both buckling load and in-plane stiffness.

From this study, it is seen that the largest number of tows with the smallest width yields the minimum gap and overlap area percentages within the laminate. The use of a course with 32 tows, each $3.175 \mathrm{~mm}(1 / 8 \mathrm{in})$ wide contributes to reduce the amount of defects within the laminate to onethird compared to the case of a course with 8 tows, each $12.7 \mathrm{~mm}(1 / 2 \mathrm{in})$ wide. If the effect of defects is factored in the optimization problem, we have observed that for a complete gap strategy the defect-free Pareto front shifts towards lower in-plane stiffness and buckling load, whereas for a complete overlap strategy the defect-free Pareto front moves towards higher values of in-plane stiffness and buckling load. The difference between gap and overlap modified Pareto fronts is the largest for the laminate that provides the maximum buckling load improvement and decreases as the layup becomes closer to the case of maximum in-plane stiffness. The layups of the three optimum Pareto solutions are different from each other, except for the two anchor points.

The results presented in this paper can be used in a future work to propose a knock-down factor for the structural properties of the variable stiffness laminates. Moreover, the number of tows and the tow width in each course can be included as design variables in the optimization problem along with the design parameters.

\section{Acknowledgement}

The authors would like to acknowledge the financial support provided by the Natural Sciences and

Engineering Research Council of Canada (NSERC) and Consortium for Research and Innovation in Aerospace in Québec (CRIAQ). We also thank the support of the National Research Council of Canada, Bombardier Aerospace and Composites Atlantic. 


\section{References:}

[1] Hyer M, Charette R. Use of curvilinear fiber format in composite structure design. AIAA journal. 1991;29:1011-5.

[2] Hyer M, Lee H. The use of curvilinear fiber format to improve buckling resistance of composite plates with central circular holes. Composite Structures. 1991;18:239-61.

[3] Gürdal Z, Olmedo R. In-plane response of laminates with spatially varying fiber orientations: variable stiffness concept. AIAA journal. 1993;31:751-8.

[4] Olmedo R, Gürdal Z. Buckling response of laminates with spatially varying fiber orientations. 34th AIAA Structures, Structural Dynamics and Materials Conference. La Jolla, CA, USA: AIAA; 1993. p. 2261-9.

[5] Gürdal Z, Tatting BF, Wu CK. Variable stiffness composite panels: effects of stiffness variation on the in-plane and buckling response. Composites Part A: Applied Science and Manufacturing. 2008;39:911-22.

[6] Akhavan H, Ribeiro P. Natural modes of vibration of variable stiffness composite laminates with curvilinear fibers. Composite Structures. 2011;93:3040-7.

[7] Setoodeh S, Abdalla MM, Gürdal Z. Design of variable-stiffness laminates using lamination parameters. Composites Part B: Engineering. 2006;37:301-9.

[8] Setoodeh S, Abdalla M, Ijsselmuiden S, Gürdal Z. Design of variable-stiffness composite panels for maximum buckling load. Composite Structures. 2009;87:109-17.

[9] Lund E, Kühlmeier L, Stegmann J. Buckling optimization of laminated hybrid composite shell structures using discrete material optimization. Citeseer; 2005.

[10] Arian Nik M, Fayazbakhsh K, Pasini D, Lessard L. Surrogate-based multi-objective optimization of a composite laminate with curvilinear fibers. Composite Structures. 2012;94:230613.

[11] Alhajahmad A AM, Gurdal Z. Optimal Design of Tow-Placed Fuselage Panels for Maximum Strength with Buckling Considerations. Journal of Aircraft. 2010;47:775-82.

[12] Blom AW, Stickler PB, Gürdal Z. Optimization of a composite cylinder under bending by tailoring stiffness properties in circumferential direction. Composites Part B: Engineering. 2010;41:157-65.

[13] Tatting BF, Gürdal Z. Automated finite element analysis of elastically-tailored plates. NASA contractor report no NASA/CR-2003-212679. 2003.

[14] Wu KC, Gürdal Z, Starnes Jr J. Structural response of compression-loaded, tow-placed, variable stiffness panels. Proceedings of the AIAA/ASME/ASCE/AHS/ASC 43rd Structures, Structural Dynamics and Materials Conference. Denver, CO, USA: AIAA; 2002.

[15] Lopes C, Gürdal Z, Camanho P. Variable-stiffness composite panels: buckling and first-ply failure improvements over straight-fibre laminates. Computers \& Structures. 2008;86:897-907.

[16] Tatting BF, Gürdal Z. Design and manufacture of elastically tailored tow placed plates. NASA contractor report no NASA/CR-2002-211919. 2002.

[17] Lopes CS, Camanho PP, Gürdal Z, Tatting BF. Progressive failure analysis of tow-placed, variable-stiffness composite panels. International Journal of Solids and Structures. 2007;44:8493516.

[18] Blom AW, Lopes CS, Kromwijk PJ, Gürdal Z, PP C. A theoretical model to study the influence of tow-drop areas on the stiffness and strength of variable-stiffness laminates. Journal of Composite Materials. 2009;43:403-25. 
[19] Fayazbakhsh K, Arian Nik M, Pasini D, Lessard L. Defect layer method to capture effect of gaps and overlaps in variable stiffness laminates made by Automated Fiber Placement. Composite Structures. 2013;97:245-51. 